SNUTP 99-015

\title{
Localization instability in the rotating D-branes
}

\author{
Rong-Gen Cai and Dahl Park \\ Center for Theoretical Physics, Seoul National University, Seoul 151-742, Korea \\ Kwang-Sup Soh \\ Department of Physics Education, Seoul National University, Seoul 151-742, Korea
}

\begin{abstract}
In the microcanonical ensemble for string theory on $A d S_{m} \times S^{n}$, there is a phase transition between a black hole solution extended over the $S^{n}$ and a solution localized on the $S^{n}$ if the $A d S_{m}$ has the topology $R^{2} \times S^{m-2}$. The phase transition will not appear if the $A d S_{m}$ has the topology $R^{2} \times T^{m-2}$, that is, when the $A d S_{m} \times S^{n}$ geometry is regarded as arising from the nearhorizon limit of a black $m-2$ brane. In this paper, we argue that when the black branes are rotating, the localization phase transition will occur between some rotating branes and corresponding Kerr black holes when the angular momentum reaches its critical value.
\end{abstract}


The Maldacena's conjecture [1] asserts that string/M theory on anti-de Sitter (AdS) space (times a compact space) is dual to certain large $N$ conformal field theory living on the boundary of the AdS (for a recent review see [2]). Thus some questions concerning large $N$ gauge theories may be answered via supergravity. Indeed, one can use this duality to calculate the correlation functions of gauge theory [3, [4], and to study thermodynamics and phase structure of the strong coupling gauge theory [5 [13].

Recently, Banks, Douglas, Horowitz and Martinec [14] argued that in the microcanonical ensemble for string theory on $A d S_{m} \times S^{n}$, there is a phase transition between a black hole solution extended on the $S^{n}$ and a solution localized on the $S^{n}$, which is associated with the Gregory-Laflamme instability (localization instability) of black strings and $p$-branes 115 . This phase transition arises as follows. It was shown by Hawking and Page [16], that a large mass Schwarzschild black hole in AdS space has positive heat capacity, while a small mass black hole has negative heat capacity. This implies that in the canonical ensemble the small mass black hole is unstable. However, in the microcanonical ensemble this instability is absent. Instead the system enters into a new phase, in which the black hole localizes on the sphere $S^{n}$, because the Schwarzschild black hole in $m+n$ dimensions has larger entropy than the equal mass Schwarzschild-AdS black hole. Thus, at higher energies, the typical state is a Schwarzschild-AdS black hole with a constant radius sphere $S^{n}$; at lower energies, where the horizon radius is smaller than the cosmological scale, the typical state becomes a Schwarzschild black hole in $m+n$ dimensions. That is, the black hole is localized on the sphere $S^{n}$.

More recently, Peet and Ross [17 found that there is a subtlety in the above localization phase transition. They showed that the existence of the phase transition crucially depends on the spatial boundary condition (topology) of the $A d S_{m}$ space. The above transition may happen when the $A d S_{m}$ has topology $R^{2} \times S^{m-2}$. If one thinks of the $A d S_{m} \times S^{n}$ geometry as arising from the near-horizon limit of some $m-2$ brane, the $A d S_{m}$ space has topology $R^{2} \times T^{m-2}$ or its large volume limit $R^{2} \times R^{m-2}$. In this case. this localization phase transition will not occur: at lower energies, the state is still the Schwarzschild-AdS black hole. This is associated with the fact that in contrast to the usual Schwarzschild-AdS black hole, the so-called topological black holes in AdS space always have positive heat capacity [18]. This implies that this localization instability does not appear as well for other higher genus topological manifold $\Sigma_{g}^{m-2}$ such that the $A d S_{m}$ has topology $R^{2} \times \Sigma_{g}^{m-2}$.

As is well known, the near-horizon geometries of the D3-brane, D1-D5-brane, M2-brane and M5-brane typically have structures $A d S_{5} \times S^{5}, A d S_{3} \times S^{3}, A d S_{4} \times S^{7}$ and $A d S_{7} \times S^{4}$, and the $A d S_{m}$ has the topology $R^{2} \times T^{m-2}$. Therefore, the localization phase transition does not take place on these branes. In the present paper, we are going to consider minor extension of them, that is, rotating D3-branes, M5-branes and M2-branes. In [19] Gubser showed that the low energy excitation of the rotating black D3-branes is thermodynamically stable up to a critical angular momentum density and a field theory model can correctly predict this critical limit. In a previous paper, in the canonical ensemble and grand canonical ensemble we investigated this critical behavior in the rotating D3-branes, M2-branes and M5-branes and found that the their heat capacity will become negative when the angular momentum exceeds a certain critical value [20]. This leads us to suspect that in the microcanonical ensemble the above localization phase transition may occur in the rotating branes. Indeed, as we will show shortly, at the low energy, but large angular momentum regime, a typical 
state should be a Kerr black hole, which has a larger entropy than the corresponding rotating black brane.

Let us begin our discussion with the rotating black D3-brane in the type IIB supergravity. The rotating black D3-brane solution has been given in [21 24]. In general, the black D3brane solution may have three angular momentum parameters. For our purpose, however, it is sufficient to consider that only a single angular momentum parameter does not vanish. In this case, The metric is

$$
\begin{aligned}
d s^{2} & =\frac{1}{\sqrt{f}}\left(-h d t^{2}+d x_{1}^{2}+d x_{2}^{2}+d x_{3}^{2}\right)+\sqrt{f}\left[\frac{d r^{2}}{\tilde{h}}-\frac{4 m l \cosh \alpha}{r^{4} \triangle f} \sin ^{2} \theta d t d \phi\right. \\
& +r^{2}\left(\triangle d \theta^{2}+\tilde{\triangle} \sin ^{2} \theta d \phi^{2}+\cos ^{2} \theta d \Omega_{3}^{2}\right]
\end{aligned}
$$

where

$$
\begin{aligned}
& f=1+\frac{2 m \sinh ^{2} \alpha}{r^{4} \triangle}, \\
& \triangle=1+\frac{l^{2} \cos ^{2} \theta}{r^{2}} \\
& \tilde{\triangle}=1+\frac{l^{2}}{r^{2}}+\frac{2 m l^{2} \sin ^{2} \theta}{r^{6} \triangle f}, \\
& h=1-\frac{2 m}{r^{4} \triangle} \\
& \tilde{h}=\frac{1}{\triangle}\left(1+\frac{l^{2}}{r^{2}}-\frac{2 m}{r^{4}}\right) .
\end{aligned}
$$

The extremal limit of the solution is approached by letting $m \rightarrow 0$. An interesting point of the solution is that the extremal limit of the solution (11) can be interpreted as some superposition of $N$ static D3-branes, rather than that of $N$ coinciding rotating D3-branes [22,25]. The near-horizon limit of static D3-branes has the geometry $A d S_{5} \times S^{5}$ with same radii $b_{3}^{2}=l_{s}^{2}\left(4 \pi g_{s} N\right)^{1 / 2}$ for the $A d S_{5}$ and $S^{5}$, where $l_{s}$ and $g_{s}$ are string length and coupling constant, respectively. For the rotating D3-brane solution (11), the geometry $A d S_{5} \times S^{5}$ is twisted because of the rotation. Near the extremal limit, some thermodynamic quantities of the black three-branes was given in [20]:

$$
\begin{aligned}
& E=3 \pi^{3} \kappa^{-2} m L^{3}, \\
& J=\pi^{7 / 4} \kappa^{-3 / 2} N^{1 / 2} m^{1 / 2} l L^{3}, \\
& \Omega=\pi^{5 / 4} \kappa^{-1 / 2} N^{-1 / 2} m^{-1 / 2} l r_{+}^{2}, \\
& T=2^{-1} \pi^{1 / 4} \kappa^{-1 / 2} N^{-1 / 2} m^{-1 / 2}\left(2 r_{+}^{3}+l^{2} r_{+}\right), \\
& S=2 \pi^{11 / 4} \kappa^{-3 / 2} N^{1 / 2} m^{1 / 2} r_{+} L^{3} .
\end{aligned}
$$

Here $E$ denotes the energy above the extremality which equals the ADM mass of the black three-brane minus the mass of the corresponding extremal one; $L^{3}$ is the spatial volume of the world volume; $2 \kappa^{2}=(2 \pi)^{7} g_{s}^{2} l_{s}^{8} ; N$ is the number of the coincident D3-branes; and $J, \Omega$, $T$ and $S$ represent the angular momentum, angular velocity, Hawking temperature, and the entropy, respectively. Finally, $r_{+}$is the horizon radius of the black three-branes, which is 


$$
r_{+}^{2}=\frac{1}{2}\left(\sqrt{l^{4}+8 m}-l^{2}\right)
$$

In [20] we showed that in the grand canonical ensemble the heat capacity becomes negative when $l^{4} / m>8 / 3$, which indicates there is a critical point at $l^{4} / m=8 / 3$. In the canonical ensemble there is also a similar critical point. Therefore the black three-brane is unstable when the angular momentum exceeds a certain critical value. According to the Maldacena's conjecture, this also implies that there is a phase transition in the $\mathcal{N}=4$ large $N$ super Yang-Mills theory in four dimensions at finite temperature [19,20]. In the microcanonical ensemble, where the energy $E$ and the angular momentum $J$ are fixed, however, similar to the case for the small mass Schwarzschild-AdS black hole, the above instability is absent. Instead a localization instability mentioned above will appear. That is, at low energy and large angular momentums, that is, when the horizon radius is smaller than the cosmological scale, the system enters into another entropically favored state, which, according to the no-hair theorem of black holes, should be described by a Kerr black hole. In addition, the D3-branes distributed on a planar disc 22 might be an appropriate source for the Kerr solution.

A D-dimensional ( $D \geq 4$ ) Kerr black hole solution with an angular momentum parameter was given in [26]. In "Boyer-Lindquist" coordinates, its metric is

$$
\begin{aligned}
d s^{2}= & -d t^{2}+\sin ^{2} \theta\left(r^{2}+a^{2}\right) d \phi^{2}+\triangle\left(d t+a \sin ^{2} d \phi^{2}\right)^{2} \\
& +\Psi d r^{2}+\rho^{2} d \theta^{2}+r^{2} \cos ^{2} \theta d \Omega_{D-4}^{2}
\end{aligned}
$$

where

$$
\begin{aligned}
& \triangle=\frac{\mu}{r^{D-5} \rho^{2}}, \quad \rho^{2}=r^{2}+a^{2} \cos ^{2} \theta, \\
& \Psi=\frac{r^{D-5} \rho^{2}}{r^{D-5}\left(r^{2}+a^{2}\right)-\mu} .
\end{aligned}
$$

This solution has the ADM mass

$$
M_{k}=\frac{(D-2) \Omega_{D-2}}{2 \kappa^{2}} \mu
$$

and the angular momentum

$$
J_{k}=\frac{\Omega_{D-2}}{\kappa^{2}} \mu a=\frac{2 a}{D-2} M_{k}
$$

Here $\Omega_{D-2}=2 \pi^{(D-1) / 2} / \Gamma[(D-1) / 2]$ is the area of a unit $(D-2)$-sphere. The horizon $r_{k}$ of the Kerr black hole is determined by the equation $g^{r r}=0$, that is,

$$
r_{k}^{D-5}\left(r_{k}^{2}+a^{2}\right)-\mu=0 .
$$

And the entropy of the solution is one quarter horizon area,

$$
S_{k}=2 \pi\left(r_{k}^{2}+a^{2}\right) r_{k}^{D-4} \Omega_{D-2} / \kappa^{2} .
$$


Here it seems worth pointing out that for the higher dimensional $(D>5)$ Kerr solutions there is no any restriction on the angular momentum in order for the solutions to have the black hole horizon.

Now Let us compare the entropies of the rotating black three brane (2) and a tendimensional Kerr black hole (8). These two entropies can be compared extactly. For small mass and large angular momentum, we find

$$
\begin{aligned}
& S \sim \frac{b_{3}^{8}}{\kappa^{2}}\left(\frac{\kappa^{2} J}{b_{3}^{8}}\right)^{-1}\left(\frac{\kappa^{2} M}{b_{3}^{7}}\right)^{3 / 2}, \\
& S_{k} \sim \frac{b_{3}^{8}}{\kappa^{2}}\left(\frac{\kappa^{2} J_{k}}{b_{3}^{8}}\right)^{-2 / 5}\left(\frac{\kappa^{2} M_{k}}{b_{3}^{7}}\right)^{8 / 5},
\end{aligned}
$$

where following [17 we have rescaled the energy of excitations $E$ as $M=L E / b_{3}$. From the above we can see that these two entropies are comparable when $\kappa^{2} M \sim b_{3}^{7}$ and $\kappa^{2} J \sim b_{3}^{8}$. Note that in this comparison we should take $M=M_{k}$ and $J=J_{k}$. When $\kappa^{2} M \sim b_{3}^{7}$, for large angular momentum $\kappa^{2} J>b_{3}^{8}$, the Kerr black hole has larger entropy than the rotating D3-brane. Therefore, a localization phase transition may happen when $\kappa^{2} M \sim b_{3}^{7}$ and $\kappa^{2} J \sim b_{3}^{8}$. In the phase diagram of $J$ versus $M$, the critical line of the localization phase transition is $\left(\frac{\kappa^{2} J}{b_{3}^{8}}\right)^{6}\left(\frac{\kappa^{2} M}{b_{3}^{7}}\right) \sim 1$. The phase above the critical line is described by the rotating D3-branes, while the phase below the line by the Kerr black hole. Of course, it should be pointed out here that there are other phases in this phase diagram, which will be discussed at the end of the paper.

Now we discuss the rotating M5-branes in the eleven-dimensional supergravity. Similar to the case of the rotating D3-brane, we also consider the case in which an angular momentum parameter does not vanish only. The rotating M5-brane solution has been given in [27,28]. The metric can be written down as

$$
\begin{aligned}
d s_{11}^{2} & =f^{-\frac{1}{3}}\left(-h d t^{2}+d x_{1}^{2}+\cdots+d x_{5}^{2}\right)+f^{\frac{2}{3}}\left[\frac{d r^{2}}{\tilde{h}}+r^{2}\left(\triangle d \theta^{2}+\tilde{\triangle} \sin ^{2} \theta d \phi^{2}\right.\right. \\
& \left.\left.+\cos ^{2} \theta d \Omega_{2}^{2}\right)-\frac{4 m l \cosh \alpha}{r^{3} \triangle f} \sin ^{2} \theta d t d \phi\right]
\end{aligned}
$$

where

$$
\begin{aligned}
& f=1+\frac{2 m \sinh ^{2} \alpha}{r^{3} \triangle}, \\
& \triangle=1+\frac{l^{2} \cos ^{2} \theta}{r^{2}}, \\
& \tilde{\triangle}=1+\frac{l^{2}}{r^{2}}+\frac{2 m l^{2} \sin ^{2} \theta}{r^{5} \triangle f}, \\
& h=1-\frac{2 m}{r^{3} \triangle}, \\
& \tilde{h}=\frac{1}{\triangle}\left[1+\frac{l^{2}}{r^{2}}-\frac{2 m}{r^{3}}\right] .
\end{aligned}
$$

The horizon of the rotating black M5-branes is determined by the equation 


$$
r_{+}^{3}+l^{2} r_{+}-2 m=0
$$

Near the extremal limit, some thermodynamic quantities of the black M5-branes are [20]

$$
\begin{aligned}
& E=20 \pi^{2} 3^{-1} \kappa^{-2} m L^{5}, \\
& J=2^{7 / 3} 3^{-1} \pi^{7 / 6} \kappa^{-5 / 3} N^{1 / 2} m^{1 / 2} l L^{5}, \\
& \Omega=2^{2 / 3} \pi^{5 / 6} \kappa^{-1 / 3} N^{-1 / 2} m^{-1 / 2} l r_{+}, \\
& T=2^{-4 / 3} \pi^{-1 / 6} \kappa^{-1 / 3} N^{-1 / 2} m^{-1 / 2}\left(3 r_{+}^{2}+l^{2}\right), \\
& S=2^{10 / 3} 3^{-1} \pi^{13 / 6} \kappa^{-5 / 3} N^{1 / 2} m^{1 / 2} r_{+} L^{5},
\end{aligned}
$$

where $2 \kappa^{2}=(2 \pi)^{8} l_{p}^{9}$ and $l_{p}$ is the eleven-dimensional Planck constant. In the case of static M5-branes, the radius of $A d S_{7}$ is $b_{5}=2 l_{p}(\pi N)^{1 / 3}$. Under the large angular momentum approximation, the entropy (12) of the rotating M5-branes behaves as

$$
S \sim \frac{b_{5}^{9}}{\kappa^{2}}\left(\frac{\kappa^{2} J}{b_{5}^{9}}\right)^{-2}\left(\frac{\kappa^{2} M}{b_{5}^{8}}\right)^{5 / 2}
$$

while a eleven-dimensional, large angular momentum Kerr black hole has entropy behavior:

$$
S_{k} \sim \frac{b_{5}^{9}}{\kappa^{2}}\left(\frac{\kappa^{2} J_{k}}{b_{5}^{9}}\right)^{-1 / 3}\left(\frac{\kappa^{2} M_{k}}{b_{5}^{8}}\right)^{3 / 2} .
$$

When $M=M_{k}$ and $J=J_{k}$, obviously, these two entropies are comparable as well if $\kappa^{2} M \sim b_{5}^{8}$ and $\kappa^{2} J \sim b_{5}^{9}$. When $\kappa^{2} M \sim b_{5}^{8}$, for larger angular momentum $\kappa^{2} J>b_{5}^{9}$, the Kerr black hole has larger entropy than the rotating M5-branes; when keeping $\kappa^{2} J \sim b_{5}^{9}$, and lowering the mass such that $\kappa^{2} M<b_{5}^{8}$, we can also see that the Kerr black hole may have larger entropy than the rotating M5-branes. We therefore conclude that the localization phase transition may happen in the rotating M5-branes when $\kappa^{2} M \sim b_{5}^{8}$ and $\kappa^{2} J \sim b_{5}^{9}$. That is, at low energies, beyond a critical angular momentum, the Kerr black hole is the entropically favored phase. In this case, the critical line is $\left(\frac{\kappa^{2} J}{b_{5}^{9}}\right)^{-5 / 3}\left(\frac{\kappa^{2} M}{b_{5}^{8}}\right) \sim 1$. Above the line is the Kerr black hole phase and the phase below the line is described by the M5-branes. Once again, there are also other phases in this phase diagram.

We now turn to the rotating black M2-branes. The rotating black M2-brane metric with a non-vanishing angular momentum is [27]

$$
\begin{aligned}
d s_{11}^{2} & =f^{-2 / 3}\left(-h d t^{2}+d x_{1}^{2}+d x_{2}^{2}\right)+f^{1 / 3}\left[\frac{d r^{2}}{\tilde{h}}+r^{2}\left(\triangle d \theta^{2}+\tilde{\triangle} \sin ^{2} \theta d \phi^{2}\right.\right. \\
& \left.\left.+\cos ^{2} \theta d \Omega_{5}^{2}\right)-\frac{4 m l \cosh \alpha}{r^{6} \triangle f} \sin ^{2} \theta d t d \phi\right]
\end{aligned}
$$

where

$$
\begin{aligned}
& f=1+\frac{2 m \sinh ^{2} \alpha}{r^{6} \triangle}, \\
& \triangle=1+\frac{l^{2} \cos ^{2} \theta}{r^{2}},
\end{aligned}
$$




$$
\begin{aligned}
& \tilde{\triangle}=1+\frac{l^{2}}{r^{2}}+\frac{2 m l^{2} \sin ^{2} \theta}{r^{8} \triangle f}, \\
& h=1-\frac{2 m}{r^{6} \triangle} \\
& \tilde{h}=\frac{1}{\triangle}\left(1+\frac{l^{2}}{r^{2}}-\frac{2 m}{r^{6}}\right) .
\end{aligned}
$$

This solution has horizon $r_{+}$given by

$$
r_{+}^{6}+r_{+}^{4} l^{2}-2 m=0
$$

In this case, near the extremal limit, we have the following thermodynamic behavior of the rotating black M2-brane [20]:

$$
\begin{aligned}
& E=3^{-1} 4 \pi^{4} \kappa^{-2} m L^{2}, \\
& J=2^{2 / 3} 3^{-1} \pi^{7 / 3} \kappa^{-4 / 3} N^{1 / 2} m^{1 / 2} l L^{2}, \\
& \Omega=2^{-2 / 3} \pi^{5 / 3} \kappa^{-2 / 3} N^{-1 / 2} m^{-1 / 2} l r_{+}^{4}, \\
& T=2^{-5 / 3} \pi^{2 / 3} \kappa^{-2 / 3} N^{-1 / 2} m^{-1 / 2} r_{+}^{3}\left(3 r_{+}^{2}+2 l^{2}\right), \\
& S=2^{5 / 3} 3^{-1} \pi^{10 / 3} \kappa^{-4 / 3} N^{1 / 2} m^{1 / 2} r_{+} L^{2} .
\end{aligned}
$$

Under the low energy and large angular momentum approximation, from ([17) we have

$$
S \sim \frac{b_{2}^{9}}{\kappa^{2}}\left(\frac{\kappa^{2} J}{b_{2}^{9}}\right)^{-1 / 2}\left(\frac{\kappa^{2} M}{b_{2}^{8}}\right),
$$

where $b_{2}=l_{p}\left(2^{5} \pi^{2} N\right)^{1 / 6} / 2$ is the radius of near-horizon geometry $A d S_{4}$ of the static M2branes. Comparing with the entropy (14) of the eleven-dimensional Kerr black hole, in this case, when $\kappa^{2} M \sim b_{2}^{8}$ and $\kappa^{2} J \sim b_{2}^{9}$, we find that these two entropies are also comparable to one another. When $\kappa^{2} M \sim b_{2}^{8}$, the entropy of the Kerr black hole is larger than that of the rotating M2-branes if $\kappa^{2} J>b_{2}^{9}$. Hence we argue that the localization phase transition may also take place for the rotating M2-branes at $\kappa^{2} M \sim b_{2}^{8}$ and $\kappa^{2} J \sim b_{2}^{9}$. The critical line is $\left(\frac{\kappa^{2} J}{b_{2}^{9}}\right)\left(\frac{\kappa^{2} M}{b_{2}^{8}}\right)^{3} \sim 1$. In this case, above the line is the M2-brane phase and the Kerr black hole describes the phase below the line.

In summary, we have discussed the microcanonical phases on the rotating D3-, M5-, and M2-branes, paying attention to the so-called localization instability. At low energies, beyond a certain critical angular momentum, we have argued that the system enters into another entropically favored phase: the phase is described by a high dimensional Kerr black hole. That is, there is a localization phase transition from some rotating black branes to corresponding Kerr black holes when the angular momentum reaches its critical value. Note that the localization transition does not occur for the static D-branes and M-branes [17]. Therefore, this is completely an effect of angular momentum. Here we should mention that for the rotating D1-D5-branes, Martinec and Sahakian |12] have already argued the existence of such kind of localization phase transition from $A d S_{3} \times S^{3}$ to a six-dimensional (6D) black hole, which is $6 \mathrm{D}$ Schwarzschild black hole spinning on the $S^{3}$. Angular momentum is introduced in this phase by spinning up the hole along an orbit on the equator of $S^{3}$ 
with momentum $P \sim J / b$, where $b$ denotes the cosmological radius of the $A d S_{3}$. It is interesting to notice the difference between the rotating D1-D5-brane system and the rotating branes considered in this paper. The near-horizon limit of the rotating D1-D5-branes is the rotating BTZ black hole. The latter is always thermodynamically stable and has a restriction on the angular momentum with $J \leq M b$, beyond which the BTZ solution does not represent a black hole. From [19,20], we know that the rotating D3-, M5- and M2-branes are thermodynamically unstable for larger angular momentum and there is no any restriction on the angular momentum in order for the branes to have a horizon. Thus a non-rotating black hole with a velocity on the $S^{n}$, which has also a restriction on the angular momentum [12], might be inappropriate to describe the new phase for these rotating branes. Similar to the Schwarzschild black hole for the low energy phase of string on the $A d S_{m} \times S^{n}$, the higher dimensional Kerr black hole might be reasonable, as discussed in the above.

Of course, there exist also other phases in the microcanonical ensemble of strings on the rotating D-branes. For instance, as shown in [14], we expect that when the horizon size of the Kerr black hole falls into the regime of the string scale, the system goes into a Hagedorn, or long string phase, where the fundamental string dominates the entropy, this is associated with the correspondence principle of Horowitz and Polchinski [29]. At the lower energies and angular momentums, another new phase may occur, where a gas of gravitons propagating in a rotating AdS space dominate the entropy. It would be interesting to make a detailed analysis of the microcanonical phases of string theory on the D-branes. In addition, in this paper we have analyzed only the rotating branes with a single angular momentum parameter. We expect that similar localization instability exists in rotating branes with multiple angular momentum parameters, because their thermodynamics is similar [30].

Acknowledgments: R.G.Cai would like to thank Prof. E. Martinec for valuable correspondence and Prof. M. Yu for helpful discussion. We are grateful to the referee for very stimulating comments which help deepen our understanding of the localization phase transition in the microcanonical ensemble of string theory. This work was supported by the KOSEF through the CTP at Seoul National University. 


\section{REFERENCES}

[1] J. Maldacena, Adv. Theor. Math. Phys. 2 (1998) 231, hep-th/9711200.

[2] J.L. Pertersen, Introduction to the Maldacena Conjecture on AdS/CFT, hep-th/9902131.

[3] S.S. Gubser, I.R. Klebanov, and A.M. Polyakov, Phys. Lett. B 428 (1998) 105, hepth/9802109.

[4] E. Witten, Adv. Theor. Math. Phys. 2 (1998) 253, hep-th/9802150.

[5] N. Itzhaki, J. Maldacena, J. Sonnenschein, and S. Yankielowicz, Phys. Rev. D 58 (1998) 046004, hep-th/9802042.

[6] E. Witten, Adv. Theor. Math. Phys. 2 (1998) 505, hep-th/9803131.

[7] M. Li, JHEP 03 (1999) 004, hep-th/9807192.

[8] J. Barbon, I. Kogan, and E. Rabinovici, Nucl. Phys. B 544 (1999) 104, hep-th/9809033.

[9] S.A. Abel, J.L.F. Barbon, I.I. Kogan, and E. Rabinovici, String Thermodynamics in D-Brane Backgrounds, hep-th/9902058.

[10] M. Li, E. Martinec, and V. Sahakian, Phys. Rev. D 59 (1999) 044035, hep-th/9809061.

[11] E. Martinec and V. Sahakian, Black holes and the super Yang-Mills phase diagram 2, hep-th/9810224.

[12] E. Martinec and V. Sahakian, Black Holes and Five-brane Thermodynamics, hepth/9901135.

[13] Y.H. Gao and M. Li, Large N strong/weak coupling phase transition and the correspondence principle, hep-th/9810053.

[14] T. Banks, M.R. Douglas, G.T. Horowitz, and E. Martinec, AdS dynamics from conformal field theory, hep-th/9808016.

[15] R. Gregory and R. Laflamme, Phys. Rev. Lett. 70 (1993) 2837, hep-th/9301052.

[16] S.W. Hawking and D.N.Page, Commun. Math. Phys. 87 (1983) 577.

[17] A.W. Peet and S.F. Ross, JHEP 12 (1998) 020, hep-th/9810200.

[18] R.G. Cai and K.S. Soh, Phys. Rev. D 59 (1999) 044013, gr-qc/9808067.

[19] S.S. Gubser, Thermodynamics of spinning D3-branes, hep-th/9810225.

[20] R.G. Cai and K.S. Soh, Critical behavior in the rotating D-branes, hep-th/9812121.

[21] J.G. Russo, New Compactification of Supergravities and Large N QCD, hep-th/9808117.

[22] P. Kraus, F. Larsen, and S.P. Trivedi, JHEP 03 (1999) 003, hep-th/9811120.

[23] J.G. Russo and K. Sfetsos, Rotating D3 branes and QCD in three dimensions, hepth/9901056.

[24] M. Cvetic and D. Youm, Nucl. Phys. B 477 (1996) 449, hep-th/9605051.

[25] K. Sfetsos, JHEP 01 (1999) 015, hep-th/9811167.

[26] R.C. Myers and M.J. Perry, Ann. Phys.(N.Y.) 172 (1986) 304.

[27] M. Cvetic and D. Youm, Nucl. Phys. B 499 (1997) 253, hep-th/9612229.

[28] C. Csáki, J. Russo, K. Sfetsos, and J. Terning, Supergravity Model for $3+1$ Dimensional QCD, hep-th/9902067.

[29] G.T. Horowitz and J. Polchinski, Phys. Rev. D 55 (1997) 6189, hep-th/9612146.

[30] M. Cvetic and S.S. Gubser, Thermodynamic Stability and Phases of General Spinning Branes, hep-th/9903132 